\title{
Ossigeno terapia iperbarica e terapia a pressione negativa nel trattamento delle lesioni difficili Hyperbaric oxygen therapy and negative pressure wound therapy in the treatment
of non-healing wounds
}

Chiara Bissoni, ${ }^{1}$ Klarida Hoxha, ${ }^{1}$ Alessandro Scalise, ${ }^{2}$ Pasquale Longobardi ${ }^{1}$

${ }^{1}$ Centro Iperbarico, Ravenna; ${ }^{2}$ Università Politecnica delle Marche, Ancona, Italia

\section{RIASSUNTO}

Lo scopo di questo articolo è valutare i risultati ottenibili trattando lesioni difficili attraverso la combinazione di ossigenoterapia iperbarica (OTI) e terapia a pressione negativa (TPN). Individuare le modalità con cui queste possano agire in sinergia coadiuvandosi, al fine di ottimizzare la rigenerazione dei tessuti e favorire la guarigione come qualità e tempi più brevi. Sono stati presi in analisi i dati di tre pazienti trattati presso il Centro Iperbarico di Ravenna che presentavano ferite agli arti inferiori aperte da più di sei settimane. È stato eseguito l'assessment iniziale della ferita e applicato un approccio multi terapeutico OTI e TPN per un periodo compreso tra 3-6 settimane. I pazienti presi in analisi sono giunti a guarigione completa entro 10 settimane di trattamento rispetto alla media di presa in carico per 28 settimane degli altri pazienti trattati presso la stessa struttura (dato reale) e alla media di 12 settimane previste nelle linee guida (benchmark). Le due terapie associate hanno prodotto un esito positivo che avrebbe richiesto tempi e costi maggiori se fossero state utilizzate singolarmente.

\section{ABSTRACT}

The purpose of this work is the evaluation of the results obtaineble by treating hard to heal wounds with the combination of Hyperbaric Oxygen Therapy (HBOT) and Negative Wound Pressure Therapy (NWPT). Identify how HBOT and NWPT can act in synergy, in order to optimize tissue regeneration and promote a good quality healing and in shorter time. The study analyzes data of three patients affected, for more than six week, by lower limb wounds and treated at the Hyperbaric Center of Ravenna. The initial wound assessment was performed and a multi-therapeutic approach, HBOT and NWPT, was applied over a period of 3-6 weeks. The patients underwent to a complete healing after a maximum of 10 weeks of treatment compared to the 28-weeks average of other patients treated at the same facility (real data) and the 12-weeks average expected in the guidelines (benchmark).

Corrispondenza: Chiara Bissoni, Centro Iperbarico, Via Augusto Torre 3, 48124 Ravenna, Italia.

Tel.: +39.0544.500152- Fax: +39.0544 .500148 .

E-mail: chiarabissoni@gmail.com,

direzione@iperbaricoravenna.it

Parole chiave: Ossigenoterapia iperbarica; Terapia a pressione negativa; Lesione cutanea; Vulnologia; Hyperbaric oxygen therapy; Negative wound pressure therapy; Skin wounds; Vulnology.

Contributi: $\mathrm{CB}$, ideazione e progettazione dello studio; $\mathrm{KH}$, supervisione e coordinamento dello studio; AS, supervisione del manoscritto; PL, approvazione finale del manoscritto.

Conflitto d'interesse: gli autori dichiarano l'assenza di conflitti d'interesse.

Fondi: nessuno.

Ricevuto per la pubblicazione: 30 maggio 2018 .

Revisione ricevuta: 27 agosto 2018.

Accettato per la pubblicazione: 27 agosto 2018.

This work is licensed under a Creative Commons Attribution NonCommercial 4.0 License (CC BY-NC 4.0).

(C) Copyright C. Bissoni et al., 2018

Licensee PAGEPress, Italy

Italian Journal of Wound Care 2018; 2(3):71-75

doi:10.4081/ijwc.2018.34
The combination of the two therapies, has led to a positive result saving time and money; the individual use of them would have required more time and costs.

\section{INTRODUZIONE}

Negli ultimi venti anni la comprensione dei meccanismi biologici coinvolti nella guarigione delle ferite ha visto un progresso decisivo. Ciò significa che ora siamo in grado di prevedere la probabile sequenza di eventi che caratterizzano il processo di guarigione e di predire il tempo necessario affinché una ferita si chiuda completamente. ${ }^{1-3}$ Questo articolo presenta una serie di casi clinici in cui alla terapia a pressione negativa (TPN) viene associata l'ossigeno terapia iperbarica (OTI) al fine di ridurre i tempi di guarigione sfruttando il loro potenziale sinergico. L'introduzione della TPN negli anni ' 90 ha segnato un punto di svolta per ciò che riguarda le medicazioni avanzate..$^{4-5} \mathrm{Al} 2018 \mathrm{RCTs}$ (per un totale di 40 studi esaminati) evidenziano l'efficacia della TPN nel ridurre il tempo di guarigione, soprattutto dopo la chirurgia del piede in concomitanza alla terapia compressiva. ${ }^{6}$ La pressione negativa, applicata da questo dispositivo, promuove una guarigione attiva provocando l'aumento dell'irrorazione sanguigna locale, la riduzione dell'edema, stimolando la formazione di tessuto di granulazione e infine avvicinando i margini della ferita. Tutto ciò 
apporta una significativa riduzione dei tempi di guarigione, come supportato da alcuni documenti di consenso, i quali ne sostengono l'efficacia su una vasta gamma di ferite difficili. ${ }^{7-9}$ L'OTI si basa sul principio per cui a pressioni superiori a quella atmosferica, aumenta la disponibilità di ossigeno disciolto nell'organismo che va a contrastare situazioni di ipossia e le infezioni, soprattutto se correlata a batteri anaerobi. L'ossigeno iperbarico agisce inoltre come farmaco, inducendo fenomeni di rivascolarizzazione indiretta per vasculogenesi e come acceleratore o modulatore della produzione di monossido di azoto, grazie alla capacità dell'OTI di ripristinare la funzionalità dell'Ossido Nitrico Sintetasi endoteliale attiva solo a pressioni parziali di ossigeno superiori $30 \mathrm{mmHg} .{ }^{10,11} \mathrm{E}$ dimostrato che l'OTI permette di ridurre i casi di amputazione maggiori del piede diabetico. ${ }^{12} \mathrm{Da}$ un'analisi comparativa che esamina sia ferite post-chirurgiche compromesse che ferite secondarie a insufficienza arteriosa, è stato rilevato che la combinazione di TPN con OTI ha prodotto risultati che superano quelli ottenuti applicando singolarmente una delle due modalità terapeutiche. ${ }^{13}$ Inoltre una ricerca ha rilevato che le due terapie associate permettono di trattare un numero maggiore di pazienti a parità di risorse economiche disponibili rispetto al trattamento con una terapia convenzionale. ${ }^{14}$

\section{MATERIALI E METODI}

Per la stesura di questo articolo sono stati presi in analisi alcuni casi di pazienti trattati con combinazione di TPN e OTI presso il Centro iperbarico di Ravenna. I casi clinici sono stati selezionati in modo da seguire diverse patologie ulcerose con ferite aperte da più di 6 settimane. Sono stati seguiti 3 pazienti ( 1 donna, 2 maschi) di età compresa tra $i$ 35 e i 70 anni. Due casi presentano tra i fattori di compromissione insufficienza venosa in assenza di ischemia critica degli arti inferiori (ICAI). In prima istanza è stato eseguito la valutazione della ferita partendo dai principi del TIME e

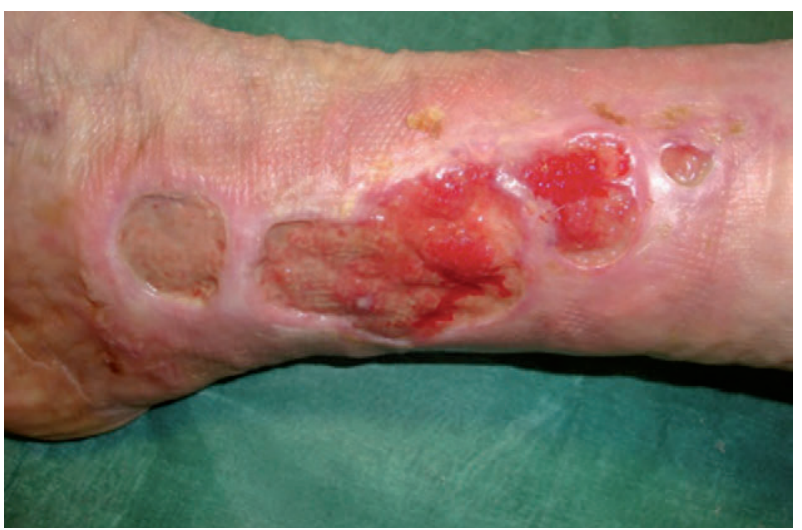

Figura 1. Caso clinico n. 1, primo accesso. applicando il Falanga Wound Bed Score e utilizzando la scala di stratificazione del rischio di amputazione WIfI, laddove appropriata. Tutti sono stati trattati con un approccio multi terapeutico comprendente wound care della ferita due volte a settimana con terapia compressiva, un ciclo da 15 a 30 sedute di OTI a cadenza giornaliera a 2,5 bar $(250 \mathrm{kPa})$ con frazione inspiratoria di ossigeno in maschera superiore al $90 \%$, per 80 minuti e applicazione di TPN per un periodo di circa 3 settimane a $-125 \mathrm{mmHg}$ in modalità continua. Quest'ultima veniva sospesa (con il clampaggio del raccordo) appena prima di entrare in camera iperbarica e ripresa subito dopo. In tutti i tre casi riportati il dispositivo TPN applicato era un dispositivo portatile (monouso e non). I due trattamenti OTI e TPN sono stati applicati in concomitanza. È bene precisare che prima di avviare il paziente all'OTI è stata attuata la valutazione dell'ossimetria transcutanea e la valutazione dell'indice caviglia-braccio e successivamente una valutazione vascolare.

Caso clinico n. 1. Donna, 67 anni. Ulcere flebolinfostatiche, in paziente con ipertensione arteriosa IVC di grado medio/severo, ICAI assente. Presenta ferite multiple al terzo medio inferiore della gamba destra, aperte da più di 8 mesi con un grado di Falanga iniziale di C2. Presenta inoltre $\mathrm{ABI}>1, \mathrm{TcpO} 2$ in tibiale posteriore $63 \mathrm{mmHg}$ e segni di infezione localizzata. L'ulcera maggiore ricopre una superficie di $13 \mathrm{~cm}^{2}$ (Figura 1).

Caso clinico n. 2. Uomo, 58 anni. Ulcera traumatica in seguito a incidente stradale, si presenta con inizio di deiscenza di ferita chirurgica versante laterale gamba sinistra in esiti di frattura pluriframmentaria biossea (pregressa ischemia traumatica acuta). Valutazione della ferita: Falanga B2, WIfI 212 che corrisponde a un alto rischio di amputazione, TcpO2 $55 \mathrm{mmHg}$ in tibiale posteriore, indice ABI non rilevabile per la presenza di ferita chirurgica. La ferita ha un'area di $22,9 \mathrm{~cm}^{2}$ e una sottominatura di $7,5 \mathrm{~cm}$, presenta inoltre infezione dei tessuti profondi con isolamento di MSSA ed esposizione tendinea (Figura 2).

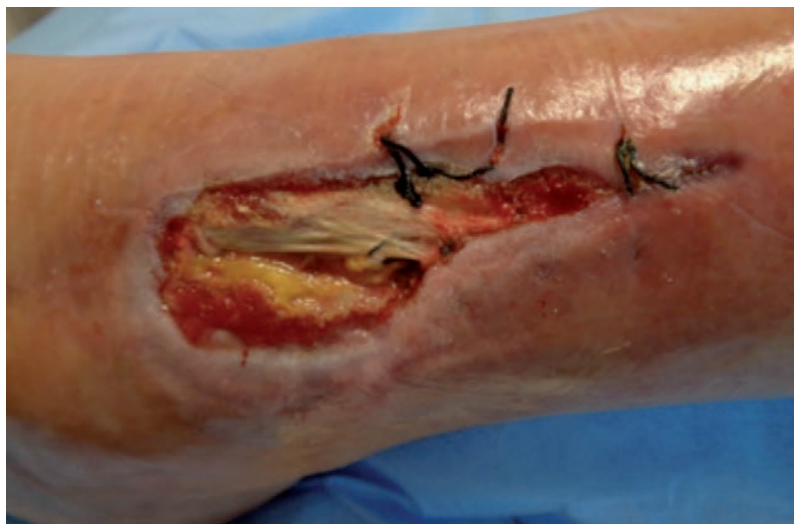

Figura 2. Caso clinico n. 2, primo accesso. 
Caso clinico n. 3. Uomo, 48 anni. Ulcere da flebolinfostasi, recidive in paziente con IVC grave, assenza di ICAI, con precedenti episodi di TVP. All'anamnesi presenta diversi fattori di compromissione tra cui ipertensione, epatite C. Quadro linfatico complesso caratterizzato da linfagite. Il paziente presenta ferite multiple all'arto sinistro. Alla valutazione si rilevano: Falanga $\mathrm{A} 3, \mathrm{ABI}>1$, TcpO2 in tibiale anteriore $34 \mathrm{mmHg}$ e segni di sovrainfezione della ferita. Si decide di mappare la superficie della lesione sovramalleolare che equivale a $10,5 \mathrm{~cm}^{2}$ (Figura 3 ).

\section{RISULTATI}

I pazienti presi in analisi sono giunti tutti a guarigione completa entro 10 settimane di trattamento. Nel primo caso: la paziente dopo 5 giorni di OTI applica la TPN (PICO: sistema monouso per la terapia a pressione negativa, Smith \& Nephew) ed esegue entrambi i trattamenti per 20 giorni. Terminato il ciclo di OTI e TPN la ferita risulta priva di infezione, granuleggiante con un grado di Falanga A1 e ridotta del $40 \%$ arrivando a misurare $7,7 \mathrm{~cm}^{2}$ (Figura 4A). Proseguendo poi con la terapia compressiva giunge a guarigione completa in otto settimane dall'inizio del trattamento (Figura 4B).

Nel secondo caso: il paziente esegue dapprima pulizia chirurgica e terapia antibiotica specifica, alla quale segue contemporaneamente un ciclo di terapia iperbarica di 20 sedute e 20 giorni di TPN (ActiV.A.C ®Therapy Unit, $\mathrm{KCI}$ ). A metà del ciclo la ferita presenta Falanga A1, riduzione della superficie di circa il 47\% $\left(12,3 \mathrm{~cm}^{2}\right)$ (Figura $5 \mathrm{~A})$. Alla fine del ciclo si prosegue con terapia compressiva e wound care avanzato fino al raggiungimento della guarigione in otto settimane totali (Figura 5B).

Nel terzo caso: il paziente viene sottoposto a un ciclo di 30 sedute OTI (1 seduta al giorno) e a terapia antibiotica mirata successiva a tampone colturale. A metà ciclo

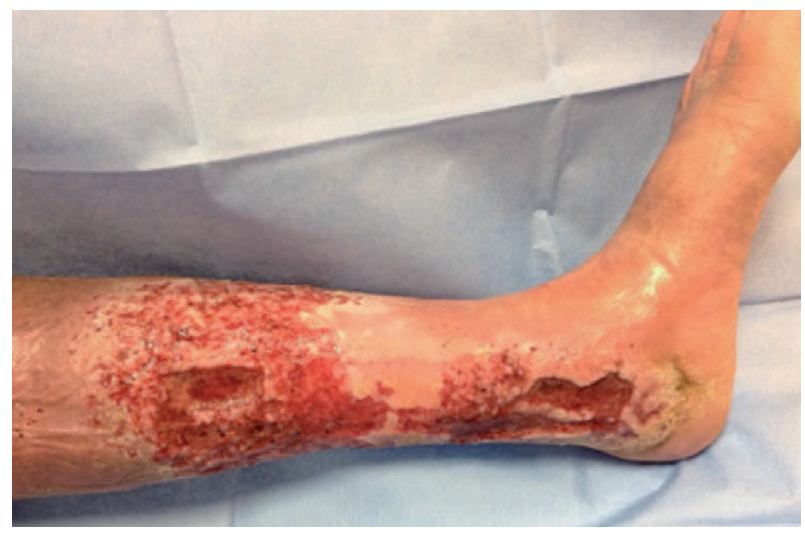

Figura 3. Caso clinico n. 3, primo accesso. si posiziona la TPN (RENASYS GO, Negative Pressure Wound Therapy, Smith \& Nephew) proseguendo per i successivi 15 giorni con entrambi in trattamenti: dopo $i$ primi 7 giorni la ferita presenta Falanga A1, riduzione della superficie del 25\% (8 $\left.\mathrm{cm}^{2}\right)$ (Figura 6A). Proseguendo con altri dieci giorni di TPN e bendaggi per le successive quattro settimane, la ferita arriva a guarigione in circa 10 settimane di trattamento (Figura 6B).

\section{DISCUSSIONE}

Alcuni studi hanno dimostrato che è possibile prevedere la guarigione valutando periodicamente determinati aspetti della ferita. ${ }^{2}$ Prendendo in esame la riduzione percentuale dell'area delle ulcere venose, è stato osservato che in circa il $77 \%$ dei casi, l'esito di guarigione poteva essere previsto su una base di una riduzione delle dimensione della ferita di oltre il $44 \%$ a tre settimane. ${ }^{2}$ Nonostante questi dati siano un'ottima guida, bisogna tener conto anche del fatto che la
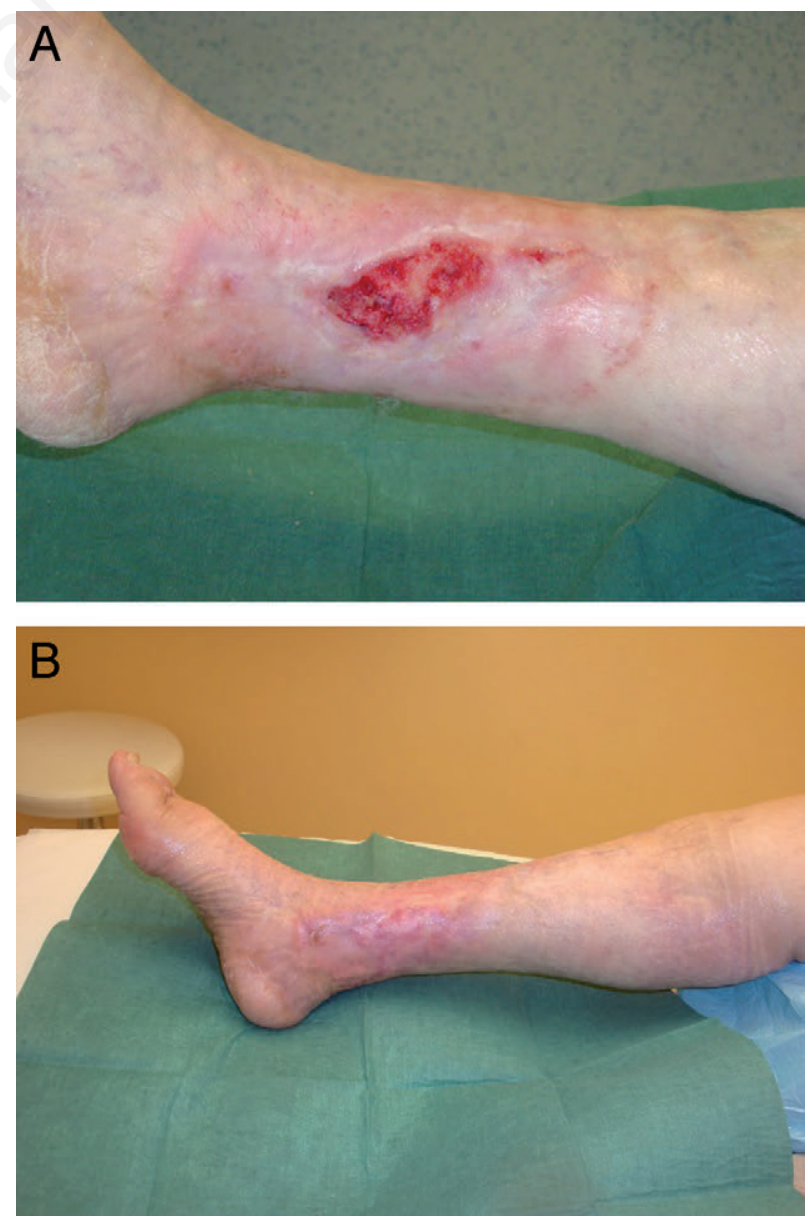

Figura 4. A) Caso clinico n. 1 dopo 3 settimane di trattamento; B) Caso clinico n. 1. Dimissione a 8 settimane. 
velocità di guarigione varia da individuo a individuo ed è influenzata da fattori intrinseci ed estrinseci, modificabili e non. Un trattamento efficace di una lesione difficile inizia con l'identificazione degli elementi che ostacolano la guarigione. Tali elementi possono essere rimossi tramite l'azione sinergica di terapie che agiscono attraverso meccanismi di azione diversi. Dai casi clinici riportati, sia in letteratura che quelli riproposti in questi case reports, si deduce che con l'ossigenoterapia iperbarica si stabilizza il tessuto e lo si protegge dalle infezioni, in contemporanea al debridement e alle medicazioni avanzate per preparare il tessuto all'applicazione della TPN. Questo approccio sinergico ha permesso il controllo del processo ischemico, infiammatorio e infettivo, che avanzava in precedenza e la rapida produzione di un letto di ferita granuleggiante, sano. Ciò significa che grazie all'OTI si possono rimuovere gli ostacoli che rallentano la riproduzione cellulare e la progressione lungo il percorso di guarigione..$^{15}$ Questo processo richiede molto tempo. Tempo in cui possono intercorrere
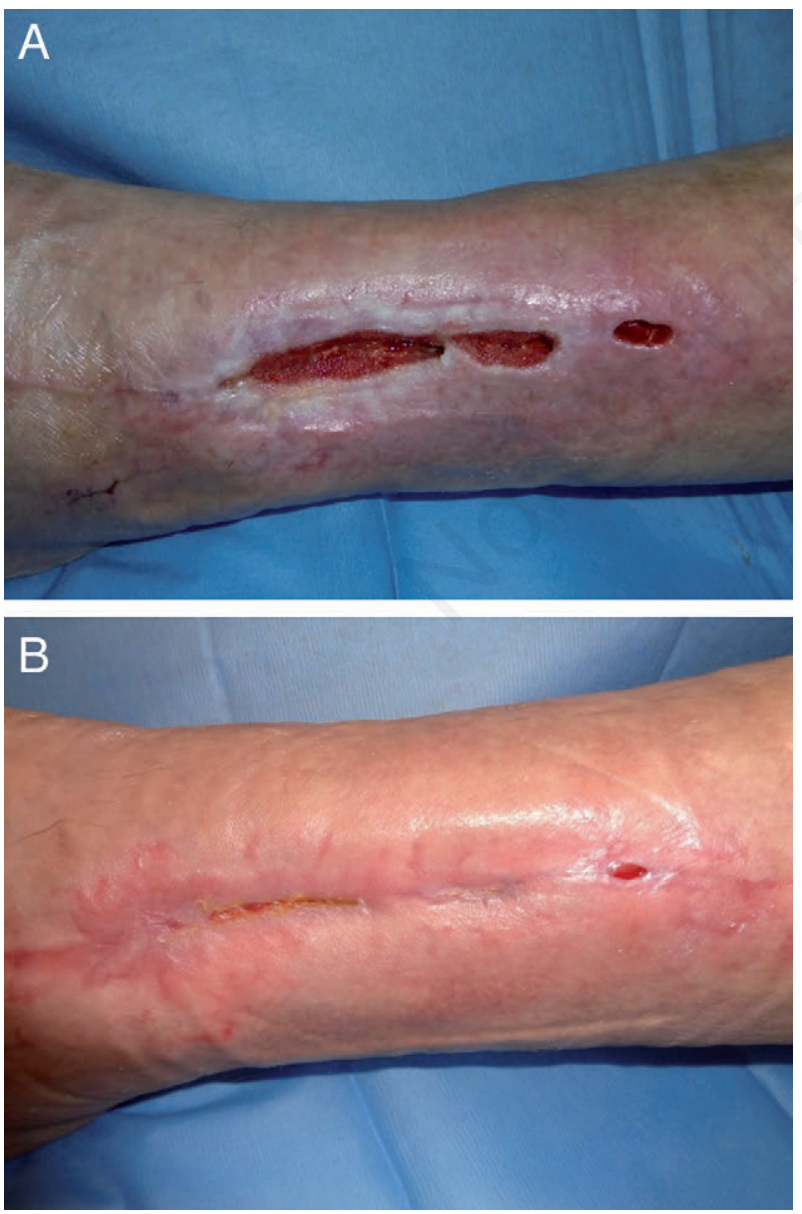

Figura 5. A) Caso clinico n. 2 dopo 3 settimane di trattamento; B) Caso clinico n. 2. Dimissione a 6 settimane. diverse complicanze, pertanto, se alla terapia iperbarica si associa una terapia come la pressione negativa, molte di queste possono essere prevenute. La TPN rimuove i fluidi in eccesso prevenendo la macerazione della cute perilesionale; rimuove con l'essudato i contaminanti che mantengono la ferita in un perenne stato infiammatorio e infine promuove la perfusione tissutale. In questo, come nella neoangiogenesi e nella riduzione della carica batterica, è sinergica con l'OTI. Laddove l'ossigeno iperbarico agisce in modo sistemico, la TPN agisce in modo locale e meccanico. Essendo inoltre l'ipossia il principale fattore patofisiologico di innesti compromessi, l'unione di OTI e TPN, per la sua azione riossigenante, favorisce l'attecchimento degli stessi al letto di ferita. Su un letto povero di ossigeno, le cellule dell'innesto tendono a morire, mentre l'iperossia prodotta dall'OTI contribuisce a migliorare la conservazione del trapianto e l'attecchimento. ${ }^{16}$ Sugli innesti cutanei trova indicazione anche la TPN, andando così a potenziarsi con l'OTI nel sfruttare al meglio le potenzialità di questi. Inoltre, in riferimento ai pazienti diabetici che diventano spesso un dilemma chirurgico a causa dalla scarsa vascolarizzazione della ferita, un approccio multidisciplinare come quello descritto può ridurre i casi di amputazione. ${ }^{17}$ Infine in letteratura emerge che l'associazione delle due te-
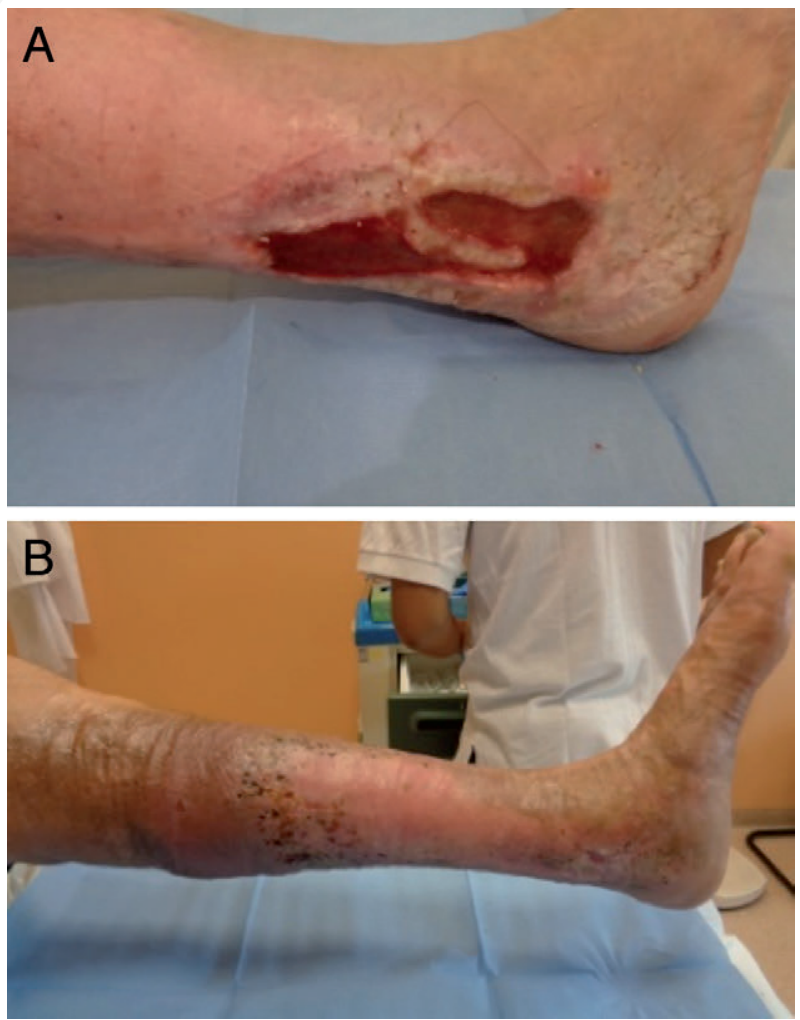

Figura 6. A) Caso clinico n. 3 dopo 4 settimane di trattamento; B) Caso clinico n. 3. Dimissione a 10 settimane. 
rapie favorisce la riduzione dei tempi di guarigione, l'abbattimento della carica batterica, l'aumento della sopravvivenza dell'innesto cutaneo ove sia stato posizionato e, spesso, la riduzione del numero di sedute iperbariche necessarie rispetto a quelle inizialmente previste. ${ }^{18}$

\section{CONCLUSIONI}

L'obiettivo sfidante che ci si pone verso tutti i pazienti al centro iperbarico di Ravenna è raggiungere la guarigione nelle 12-16 settimane. Nell'anno 2017 la media di presa in carico dei pazienti si aggira, in realtà, intorno alle 28 settimane. Considerando i casi selezionati, si rileva che l'applicazione delle due terapie in contemporanea o consequenzialmente ha prodotto una riduzione delle superfici di ferita in media del $40 \%$ al termine del ciclo di OTI e avviato il processo di guarigione. Successivamente, con wound care avanzato e terapia compressiva, si è ottenuta la guarigione completa in media tra le 6 e le 12 settimane di trattamento. Le due terapie associate hanno prodotto un risultato positivo che avrebbe richiesto tempi maggiori se fossero state utilizzate singolarmente. Nonostante gli ambiti di azioni siano simili, i meccanismi d'azione sono diversi e non si sovrappongono ma si implementano amplificando l'azione di entrambe le terapie, facilitando il raggiungimento della guarigione. Tenendo conto di quanto sopra scritto appare ragionevole ipotizzare che l'aumento dei costi nell'immediato per l'associazione di due terapie sia giustificato dalla ottimizzazione dei tempi di guarigione, dal miglioramento della qualità di vita del paziente. Gli Autori ritengono che l'associazione OTI - TPN rappresenti un contenimento dei costi economici rispetto all'utilizzo di terapie meno dispendiose nell'immediato ma meno efficaci e quindi più costose nel lungo termine.

\section{BIBLIOGRAFIA}

1. Troxer M, Vowden K, Vowden P. Integrating adjunctive therapy into practice: the importance of recognising 'hard-toheal' wounds. World Wide Wounds 2006. Disponibile al sito: http://www.worldwidewounds.com/2006/december/Troxler/I ntegrating-Adjunctive-Therapy-Into-Practice.html

2. Phillips TJ, Machado F, Trout R, et al. Prognostic indicators in venous ulcers. J Am Acad Dermatol 2000;43:627-30.
3. Falanga V. Measurements in Wound Healing. Int J Low Extrem Wounds 2008;7:9-11.

4. Morykwas MJ, Argenta LC, Shelton-Brown EI, Mcguirt W. Vacuum-assisted closure: a new method for wound control and treatment: animal studies and basic foundation. Ann Plast Surg 1997;38:553-62.

5. Morykwas MJ, Simpson J, Punger K, et al. Vacuum-assisted closure: state of basic research and physiologic foundation. Plast Reconstr Surg 2006;11:7.

6. Armstrong DG, Lavery LA. Diabetic Foot Study Consortium. Negative pressure wound therapy after partial diabetic foot amputation: a multicentre, randomised controlled trial. Lancet 2005;366:1704-10.

7. European Wound Management Association (EWMA). Documento di posizionamento: La pressione topica negativa nella gestione delle ferite. London, UK: MEP Ltd; 2007.

8. World Union of Wound Healing Societies (WUWHS) Consensus Document. Closed surgical incision management: understanding the role of NPWT. Wounds International; 2016.

9. Venturi ML, Attinger CE, Mesbahi AN, et al. Mechanisms and clinical applications of the vacuum-assisted closure (VAC) Device: a review. Am J Clin Dermatol 2005;6:185-94.

10. Camporesi EM, Bosco G. Mechanisms of action of hyperbaric oxygen therapy. Undersea Hyperb Med 2014;41:247-52.

11. Eggleton P, Bishop AJ, Smerdon GR. Safety and efficacy of hyperbaric oxygen therapy in chronic wound management: current evidence. Chronic Wound Care Manag Res 2015;2:81-93.

12. Löndahl M, Katzman P, Nilsson A, Hammarlund C. Hyperbaric oxygen therapy facilitates healing of chronic foot ulcers in patients with diabetes. Diabets Care 2010;33:998-1003.

13. Niezgoda JA. Combining negative pressure wound therapy with other wound management modalities. Ostomy Wound Manage 2005;51:36-8.

14. Schweyer MA, Murdock B, Davis HA. Negative pressure wound therapy - positive change for an acute care hospital and a hospital based comprehensive wound care center. UHMS Annual Meeting, Salt Lake, USA; June 2008.

15. Kranke P, Bennett MH, Martyn-St James M, et al. Hyperbaric oxygen therapy for chronic wounds. Cochrane Database Syst Rev 2015;6:CD004123.

16. Zamboni WA, Roth AC, Russell RC, et al. The effect of acute hyperbaric oxygen therapy on axial pattern skin flap survival when administered during and after total ischemia. J Reconstr 2 Microsurg 1989;5:343-7.

17. Cole E. Use of Multiple adjunctive negative pressure wound therapy modalities to manage diabetic lower-extremity wounds. Eplasty 2016;16:e34.

18. Fabian TS, Kaufman HJ, Lett ED, et al. The evaluation of subatmospheric pressure and hyperbaric oxygen in ischemic full-thickness wound healing. Am Surg 2000;66:1136-43. 